\title{
CATEGORIFIED INVARIANTS AND THE BRAID GROUP
}

\author{
JOHN A. BALDWIN AND J. ELISENDA GRIGSBY
}

\begin{abstract}
We investigate two "categorified" braid conjugacy class invariants, one coming from Khovanov homology and the other from Heegaard Floer homology. We prove that each yields a solution to the word problem but not the conjugacy problem in the braid group.
\end{abstract}

\section{INTRODUCTION}

Recall that the $n$-strand braid group $B_{n}$ admits the presentation

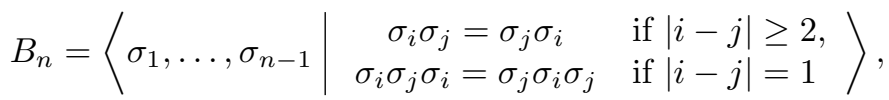

where $\sigma_{i}$ corresponds to a positive half twist between the $i$ th and $(i+1)$ st strands. Given a word $w$ in the generators $\sigma_{1}, \ldots, \sigma_{n-1}$ and their inverses, we will denote by $\sigma(w)$ the corresponding braid in $B_{n}$. Also, we will write $\sigma \sim \sigma^{\prime}$ if $\sigma$ and $\sigma^{\prime}$ are conjugate elements of $B_{n}$. As with any group described in terms of generators and relations, it is natural to look for combinatorial solutions to the word and conjugacy problems for the braid group:

(1) Word problem: Given words $w, w^{\prime}$ as above, is $\sigma(w)=\sigma\left(w^{\prime}\right)$ ?

(2) Conjugacy problem: Given words $w, w^{\prime}$ as above, is $\sigma(w) \sim \sigma\left(w^{\prime}\right)$ ?

The fastest known algorithms for solving Problems (1) and (2) exploit the Garside structure(s) of the braid group (cf. [7] for a survey and [10] for an implementation). In addition, any faithful representation of $B_{n}$ for which the images of the generators and the product rule can be described combinatorially - for example, the Lawrence-Krammer representation [24, 22, 6] - provides a solution to Problem (1).

The present work is an attempt to understand what two popular combinatorial link homology theories coming from representation theory and symplectic geometry - namely, Khovanov homology and link Floer homology - can tell us about Problems (1) and (2). Both theories are powerful enough to detect the unknot 35,23 . Can they detect the trivial braid? Can they distinguish braid conjugacy classes?

In this short note, we explain how to extract braid conjugacy class invariants from both theories and we prove that each of these invariants provides a solution to Problem (1) but not (naively) to Problem (2). The approaches to Problems (1) and (2) described here are, at present, more computationally involved than the solutions alluded to at the top. In that sense, our results are primarily of theoretical interest. Perhaps more tractable solutions to these problems can be obtained along similar lines in the future as faster algorithms are discovered for computing Khovanov and link Floer homology.

JAB was partially supported by NSF grant number DMS-1104688.

JEG was partially supported by NSF grant number DMS-0905848 and NSF CAREER award DMS1151671. 
Below, we provide a brief description of the "categorified" braid conjugacy class invariants studied in this note. Both are invariants of isotopy classes of oriented links in the solid torus complement of a neighborhood of an oriented unknot $B \subset S^{3}$. We will think of $B$ as the compactification of the oriented $z$-axis in $\mathbb{R}^{3}$,

$$
B=\{(r, \theta, z) \mid r=0\} \cup\{\infty\} \subset \mathbb{R}^{3} \cup\{\infty\}=S^{3},
$$

and the complement $S^{3}-N(B)$ as the product

$$
A \times I=\{(r, \theta, z) \mid r \in[1,2], \theta \in[0,2 \pi), z \in[0,1]\}
$$

of an annulus $A$ with the interval $I=[0,1]$.

Given a braid $\sigma \in B_{n}$ (read and oriented, by convention, from top to bottom), we will imagine its oriented closure $\widehat{\sigma}$ as living in $A \times I$ such that $\widehat{\sigma}$ intersects every disk

$$
D_{t}=\{(r, \theta, z) \mid \theta=t\} \cup\{\infty\}
$$

positively in $n$ points, where $D_{t}$ is oriented so that $B=\partial D_{t}$. Note that $\widehat{\sigma} \subset A \times I$ is welldefined up to isotopy and, moreover, that conjugate braids give rise to isotopic closures. Thus, any invariant of isotopy classes of links in $A \times I$ gives rise to a braid conjugacy class invariant.

The central objects of study in this paper are the sutured annular Khovanov homology $\operatorname{SKh}(\widehat{\sigma} \subset A \times I)($ cf. Section 2.1) and the link Floer homology $\widehat{\mathrm{HFL}}(\widehat{\sigma} \cup B)$ (cf. Section 2.2). Both are invariants of the conjugacy class of $\sigma$ per the observation above. As mentioned earlier, we will prove that both can be used to distinguish unequal braids (cf. Theorem 1 ) but that neither always distinguishes non-conjugate braids (cf. Theorem 2 and Corollary 22. These invariants share other structural features, including a relationship with the Burau representation

$$
\Psi: B_{n} \rightarrow G L_{n}\left(\mathbb{Z}\left[T^{ \pm 1}\right]\right)
$$

(cf. Remarks 2.1 and 2.3). In particular, the graded Euler characteristic of $\widehat{\mathrm{HFL}}(\widehat{\sigma} \cup$ $B$ ) is (more or less) the characteristic polynomial of $\Psi(\sigma)$. We will show, however, that the homology contains more braid information than does the polynomial in general (cf. Proposition 2.1.

It bears mentioning that the standard (i.e. non-sutured) versions of Khovanov homology and knot Floer homology can also be used to detect the trivial braid, thanks to a combination of recent work on the Khovanov/Floer side with older work of Birman-Menasco on braid foliations. Specifically, Batson-Seed [4 have shown, building on work of Hedden-Ni [18, which, in turn, builds on work of Kronheimer-Mrowka 23, that if $L_{n}$ is a link with $n$ components, then $\operatorname{Kh}\left(L_{n}\right) \cong \operatorname{Kh}\left(U_{n}\right)$ iff $L_{n}=U_{n}$, where $U_{n}$ is the $n$-component unlink. Analogously, $\mathrm{Ni} 34$ has shown, building on work of Ozsváth-Szabó [35, that $\widehat{\operatorname{HFK}}\left(L_{n}\right) \cong$ $\widehat{\operatorname{HFK}}\left(U_{n}\right)$ iff $L_{n}=U_{n}$.

To determine whether $\sigma=\mathbb{1} \in \mathfrak{B}_{n}$, it then suffices to compute $\operatorname{Kh}(\widehat{\sigma})$ (resp. $\widehat{\operatorname{HFK}}(\widehat{\sigma})$ ). If one obtains the same answer as for $U_{n}$, then the Batson-Seed (resp. Ni) result implies that $L_{n}=U_{n}$. A corollary of Birman-Menasco's main result in 8 - that an $n$-strand braid whose closure is $U_{n}$ is conjugate to the trivial braid - then tells us that $\sigma=\mathbb{1}$.

There are two primary reasons for focusing on the invariants $\widehat{\operatorname{HFL}}(\widehat{\sigma} \cup B)$ and $\operatorname{SKh}(\widehat{\sigma})$ in this paper. First, they're more intrinsically braid invariants and carry more conjugacy class information than $\widehat{\mathrm{HFK}}(\widehat{\sigma})$ and $\operatorname{Kh}(\widehat{\sigma})$, which depend only on the isotopy class of $\widehat{\sigma}$ as a link in $S^{3}$, and not at all on its embedding in $S^{3}-N(B)$. Second, and perhaps most significantly, our proof that $\operatorname{SKh}(\widehat{\sigma})$ detects the trivial braid is entirely combinatorial. It does not rely 
on Floer homology or gauge theory at all, unlike the proof that $\operatorname{Kh}(\widehat{\sigma})$ detects the trivial braid, which relies on deep results in both Heegaard and instanton Floer homology.

Finally, we note that Proposition 3.1 and Corollary 1 should be of independent interest, as they demonstrate that Plamenevskaya's invariant-while not an effective invariant of transverse links (cf. [12])-does detect interesting geometric features of braids.

Acknowledgements. The authors wish to thank Christian Blanchet, who asked the question that initiated this investigation, and Stephan Wehrli, for a number of interesting conversations. We owe a special debt of gratitude to Matt Hedden and Liam Watson, for sharing their paper [19. In it, they show (using a simple but powerful argument that arose during conversations with Jeremy Van Horn-Morris) that if $K$ is a Floer-simple fibered knot in a closed, oriented 3-manifold $Y$, then the monodromy of $K$ must be trivial. Our proofs of Theorem 1. (a) and 1.(b) were inspired by this idea. Finally, we thank Eddy Godelle for pointing us to the main result of [14.

\section{Categorified Braid Conjugacy Class Invariants}

In this section, we briefly recall the construction of sutured annular Khovanov homology and some basic features of link Floer homology. We will assume the reader is familiar with ordinary Khovanov homology and knot Floer homology. All chain complexes and homology theories considered in this paper are with coefficients in $\mathbb{E}:=\mathbb{Z} / 2 \mathbb{Z}$.

2.1. Sutured Annular Khovanov Homology. Sutured annular Khovanov homology was originally defined in [1] as a categorification of the Kauffman bracket skein module of $A \times I$. It was studied further in [40, 16, where a connection with sutured Floer homology was discovered (hence, the name). The theory associates to an oriented link $\mathbb{L} \subset A \times I$ a triply-graded vector space

$$
\operatorname{SKh}(\mathbb{L})=\bigoplus_{i, j, k} \operatorname{SKh}^{i}(\mathbb{L} ; j, k),
$$

which is an invariant of the oriented isotopy class of $\mathbb{L} \subset A \times I$.

Its construction starts with a projection of $\mathbb{L}$ onto the annulus $A \times\{1 / 2\}$. This projection may be viewed as a planar diagram $D$ in $S^{2}-\{X, O\}$, where $X$ and $O$ are basepoints in $S^{2}$ corresponding to the inner and outer boundary circles of $A \times\{1 / 2\}$. Forgetting the data of the $X$ basepoint temporarily, we may think of $D$ as a planar diagram in $\mathbb{R}^{2}=S^{2}-\{O\}$ and form the ordinary bigraded Khovanov complex

$$
\operatorname{CKh}(D)=\bigoplus_{i, j} \operatorname{CKh}^{i}(D ; j)
$$

from a cube of resolutions of $D$ in the usual way. Here, $i$ and $j$ are the homological and quantum gradings, respectively 1 The basepoint $X$ gives rise to a filtration on $\operatorname{CKh}(D)$, and $\operatorname{SKh}(\mathbb{L})$ is defined to be the $(\mathrm{co})$ homology of the associated graded object.

To define this filtration, we choose an oriented arc from $X$ to $O$ missing all crossings of the diagram $D$. As described in [17, Sec. 4.2], the generators of $\operatorname{CKh}(D)$ are in one-to-one correspondence with enhanced (i.e., oriented) resolutions. We define the " $k$ " grading of an oriented resolution to be the algebraic intersection number of this resolution with our

\footnotetext{
${ }^{1}$ The grading, $i$, is really a cohomological grading, as the Khovanov differential increases it by 1.
} 
oriented arc, up to some overall shift. Roberts proves ([40, Lem. 1]) that the Khovanov differential does not increase this extra grading. One therefore obtains a filtration,

$$
0 \subseteq \ldots \subseteq \mathcal{F}_{n-1}(D) \subseteq \mathcal{F}_{n}(D) \subseteq \mathcal{F}_{n+1}(D) \subseteq \ldots \subseteq \operatorname{CKh}(D),
$$

where $\mathcal{F}_{n}(D)$ is the subcomplex of $\operatorname{CKh}(D)$ generated by oriented resolutions with $k$ grading at most $n$. Let

$$
\mathcal{F}_{n}(D ; j)=\mathcal{F}_{n}(D) \cap \bigoplus_{i} \operatorname{CKh}^{i}(D ; j) .
$$

The sutured annular Khovanov homology groups of $\mathbb{L}$ are defined to be

$$
\operatorname{SKh}^{i}(\mathbb{L} ; j, k):=H^{i}\left(\frac{\mathcal{F}_{k}(D ; j)}{\mathcal{F}_{k-1}(D ; j)}\right) .
$$

The lemma below follows directly.

Lemma 2.1. There is a spectral sequence whose $E_{1}$ term is $S K h(\mathbb{L} \subset A \times I)$ and whose $E_{\infty}$ term (ignoring the $k$ grading) is isomorphic to $K h\left(\mathbb{L} \subset S^{3}\right)$. Moreover, the $d_{n}$ differential shifts the $(i, j, k)$ multi-grading by $(1,0,-n)$.

Given a braid $\sigma$, we will be interested in $\operatorname{SKh}(\widehat{\sigma})$, where $\widehat{\sigma} \subset A \times I$ is the oriented closure of $\sigma$ as described in the Introduction.

Remark 2.1. It is shown in 2 that the summand of $\operatorname{SKh}(\widehat{\sigma})$ in the next-to-top $k$ grading is equal to the Hochschild homology of the braid bimodule constructed by Khovanov and Seidel in [21. This bimodule detects the trivial braid [21, Cor. 1.2] (and its action categorifies the Burau representation [21, Prop. 2.8]). These facts together with Theorem 2 imply that a categorical group representation may be faithful even while its Hochschild homology is not faithful on conjugacy classes. Similar results have been obtained in the Heegaard Floer setting by Hedden and Watson [19] in combination with Lipshitz, Ozsváth and Thurston [25, Thm. 14], 26].

On the other hand, Theorem 1 suggests the following question.

Question 2.1. Suppose one has a faithful weak action, in the sense of [21, Def. 2.6], of a group $G$ on a (derived) category of modules over an $\left(A_{\infty}\right)$ algebra $A$, where the functor associated to an element $g \in G$ is given by taking a (derived) tensor product with a (derived equivalence class of ) bimodule $\mathcal{M}_{g}$. Let $H H\left(A, \mathcal{M}_{g}\right)$ denote the Hochschild homology of $A$ with coefficients in $\mathcal{M}_{g}$. Does

$$
H H\left(A, \mathcal{M}_{g}\right)=H H\left(A, \mathcal{M}_{\mathbb{1}}\right)
$$

necessarily imply that $g=\mathbb{1}$ ?

2.2. Link Floer Homology. Link Floer homology was defined in [36] as a generalization of knot Floer homology and a categorification of the multi-variable Alexander polynomial. One version of the theory associates to an oriented link $\mathbb{L} \subset S^{3}$ expressed as a union of $k$ sublinks, $\mathbb{L}=L_{1} \cup \cdots \cup L_{k}$, a graded vector space

$$
\widehat{\mathrm{HFL}}(\mathbb{L})=\bigoplus_{d, A_{L_{1}}, \ldots, A_{L_{k}}} \widehat{\operatorname{HFL}}_{d}\left(\mathbb{L} ; A_{L_{1}}, \ldots, A_{L_{k}}\right)
$$

which is an invariant of the oriented isotopy class of $\mathbb{L} \subset S^{3}$. Here, $d$ is the Maslov grading and the $A_{L_{i}}$ are the Alexander gradings associated to the sublinks $L_{i} \stackrel{2}{2}^{2}$ When $k=1, \widehat{\operatorname{HFL}}(\mathbb{L})$

\footnotetext{
${ }^{2}$ Typically, one associates an Alexander grading to each component of $\mathbb{L}$. The theory we describe here is obtained by "flattening" the multi-grading associated to each sublink $L_{i}$ into a single grading by summing.
} 
as described here is isomorphic to the knot Floer homology $\widehat{\mathrm{HFK}}(\mathbb{L})$ after an overall shift of the Maslov grading [37, Thm. 1.1].

Link Floer homology enjoys many symmetries. To begin with, $\widehat{\mathrm{HFL}}(\mathbb{L})$ is supported in Alexander multi-gradings that are symmetric about the origin in $\mathbb{R}^{k}$,

$$
\widehat{\mathrm{HFL}}_{d}\left(\mathbb{L} ; A_{L_{1}}, \ldots, A_{L_{k}}\right) \cong \widehat{\mathrm{HFL}}_{d-2 S}\left(\mathbb{L} ;-A_{L_{1}}, \ldots,-A_{L_{k}}\right),
$$

where $S=\sum_{i} A_{L_{i}}$. Moreover, if $\mathbb{L}^{\prime}$ is the link obtained by replacing $L_{i}$ with $-L_{i}$, then

$$
\widehat{\mathrm{HFL}}_{d}\left(\mathbb{L} ; A_{L_{1}}, \ldots, A_{L_{i}}, \ldots, A_{L_{k}}\right) \cong \widehat{\mathrm{HFL}}_{d-2 A_{L_{i}}+l_{i}}\left(\mathbb{L}^{\prime} ; A_{L_{1}}, \ldots,-A_{L_{i}}, \ldots, A_{L_{k}}\right),
$$

where $l_{i}=\operatorname{lk}\left(L_{i}, \mathbb{L}-L_{i}\right)$. Similarly, if $m(\mathbb{L})$ denote the mirror of $\mathbb{L}$, then

$$
\widehat{\mathrm{HFL}}_{d}\left(\mathbb{L} ; A_{L_{1}}, \ldots, A_{L_{k}}\right) \cong \widehat{\mathrm{HFL}}_{2 S-d+1-|\mathbb{L}|}\left(m(\mathbb{L}) ; A_{L_{1}}, \ldots, A_{L_{k}}\right) .
$$

See [30, Sec. 5] and [37, Sec. 8] for discussions of these symmetries.

The link Floer homology of $\mathbb{L}$ is the homology of a chain complex defined in terms of a multi-pointed Heegaard diagram for $\mathbb{L}$. In an abuse of notation, we will denote this complex by $\widehat{\mathrm{CFL}}(\mathbb{L})$. For each sublink $L_{i}, \widehat{\mathrm{CFL}}(\mathbb{L})$ can be realized as the associated graded object of a filtration on some complex $C\left(\mathbb{L}-L_{i}\right)$, where

$$
H_{*}\left(C\left(\mathbb{L}-L_{i}\right)\right) \cong \widehat{\mathrm{HFL}}\left(\mathbb{L}-L_{i}\right) \otimes V^{\otimes\left|L_{i}\right|}
$$

up to a shift of the Alexander multi-grading, for $k \geq 2$. Here, $V$ is the triply-graded vector space $\mathbb{F} \oplus \mathbb{F}$ whose summands are supported in Maslov gradings 0 and -1 and Alexander multi-grading $(0, \ldots, 0) \subset \mathbb{R}^{k-1}$. This leads to the following lemma (the claim about grading shifts follows immediately from the discussions in Subsections 3.7 and 8.1 of [37]).

Lemma 2.2. For $k \geq 2$, there is a spectral sequence whose $E_{1}$ term is $\widehat{H F L}(\mathbb{L})$ and whose $E_{\infty}$ term (ignoring the $A_{L_{i}}$ grading) is isomorphic to the vector space obtained from $\widehat{H F L}(\mathbb{L}-$ $\left.L_{i}\right) \otimes V^{\otimes\left|L_{i}\right|}$ by shifting each Alexander grading $A_{L_{j}}$ by $\frac{1}{2} l k\left(L_{j}, L_{i}\right)$.

The following describes a similar spectral sequence in the case that $k=1$ (cf. 37).

Lemma 2.3. For $k=1$, there is a spectral sequence whose $E_{1}$ term is $\widehat{H F L}(\mathbb{L})$ and whose $E_{\infty}$ term is a rank $2^{|\mathbb{L}|-1}$ vector space.

We now restrict our attention to the case $\mathbb{L}=\widehat{\sigma} \cup B$. Note that $\widehat{\mathrm{HFL}}(\widehat{\sigma} \cup B)$ is an invariant of the oriented isotopy class of $\widehat{\sigma} \subset A \times I$.

Remark 2.2. That $\widehat{\mathrm{HFL}}(\widehat{\sigma} \cup B)$ is the homology of the associated graded object of a filtration on a complex which computes $\widehat{\mathrm{HFL}}(\widehat{\sigma}) \otimes V$ (up to an Alexander grading shift) closely parallels the relationship between $\operatorname{SKh}(\widehat{\sigma} \subset A \times I)$ and $\operatorname{Kh}\left(\widehat{\sigma} \subset S^{3}\right)$ described in the previous subsection.

Remark 2.3. The fact that link Floer homology categorifies the multi-variable Alexander polynomial, combined with an older result of Morton 32, implies that $\widehat{H F L}(\widehat{\sigma} \cup B)$ categorifies the characteristic polynomial $\operatorname{det}(\lambda-\Psi(\sigma))$, where

$$
\Psi: B_{n} \rightarrow G L_{n}\left(\mathbb{Z}\left[T^{ \pm 1}\right]\right)
$$

is the Burau representation of $B_{n}$. More precisely,

$$
\sum_{d, A_{\widehat{\sigma}}, A_{B}}(-1)^{d} \cdot \operatorname{rk} \widehat{H F L}_{d}\left(\widehat{\sigma} \cup B ; A_{\widehat{\sigma}}, A_{B}\right) \cdot T^{A_{\widehat{\sigma}}} \cdot \lambda^{A_{B}}=\operatorname{det}(\lambda-\Psi(\sigma)) \cdot(T-1)^{|\widehat{\sigma}|},
$$


up to an overall factor of $\pm T^{m_{1}} \cdot \lambda^{m_{2}}$ for some $m_{1}, m_{2} \in \frac{1}{2} \mathbb{Z}$.

On the other hand, since the Burau representation is not faithful for $n \geq 5$ (cf. [31, 28, 5]), Theorem 1. (b) implies that $\widehat{\mathrm{HFL}}(\widehat{\sigma} \cup B)$ contains strictly more information about $\sigma$ than does $\operatorname{det}(\lambda-\Psi(\sigma))$ :

Proposition 2.1. For each $n \geq 5$ there exists a braid $\sigma \in B_{n}$ for which

$$
\operatorname{det}(\lambda-\Psi(\sigma))=\operatorname{det}(\lambda-\Psi(\mathbb{1})) \text { but } \widehat{H F L}(\widehat{\sigma} \cup B) \nsucceq \widehat{H F L}(\widehat{\mathbb{1}} \cup B) .
$$

\section{Trivial Braid Detection: Solution To Word Problem}

The goal of this section is to prove that $\operatorname{SKh}(\widehat{\sigma})$ and $\widehat{\operatorname{HFL}}(\widehat{\sigma} \cup B)$ detect the trivial braid and therefore provide solutions to Problem (1), per the theorem below.

Theorem 1. Suppose $\sigma \in B_{n}$.

(a) If $S K h(\widehat{\sigma}) \cong S K h(\widehat{\mathbb{1}})$, then $\sigma=\mathbb{1}$.

(b) If $\widehat{H F L}(\widehat{\sigma} \cup B) \cong \widehat{H F L}(\widehat{\mathbb{1}} \cup B)$ and $\sigma$ is a pure braid, then $\sigma=\mathbb{1}: 3$

In particular, both $S K h(\widehat{\sigma})$ and $\widehat{H F L}(\widehat{\sigma} \cup B)$ can be used to give solutions to the word problem in the braid group.

The proof of Theorem 1. (a) relies on certain properties of Plamenevskaya's invariant 39] of transverse links. We pause to discuss these first, delaying the proof of Theorem 1 to Subsection 3.2

3.1. Plamenevskaya's Invariant and Trivial Braid Detection. Let $D_{n}$ denote the standard unit disk with $n$ distinct marked points $p_{1}, \ldots, p_{n}$ positioned along the real axis.

Definition 3.1. We say an arc $\gamma:[0,1] \rightarrow D_{n}$ is admissible if it satisfies

(1) $\gamma$ is a smooth imbedding transverse to $\partial D_{n}$,

(2) $\gamma(0)=-1 \in \mathbb{C}$ and $\gamma(1) \in\left\{p_{1}, \ldots, p_{n}\right\}$,

(3) $\gamma(t) \in D_{n} \backslash\left(\partial D_{n} \cup\left\{p_{1}, \ldots, p_{n}\right\}\right)$ for all $t \in(0,1)$, and

(4) $\frac{d \gamma}{d t} \neq 0$ for all $t \in[0,1]$.

Note that the above definition differs from the one given in [3, Sec. 4], where admissible arcs originate and terminate on $\partial D_{n}$. We will often abuse notation and use $\gamma$ to refer to the image of $\gamma$ in $D_{n}$.

Definition 3.2. Two admissible arcs $\gamma, \gamma^{\prime}$ are said to be pulled tight if they satisfy one of:

- $\gamma=\gamma^{\prime}$, or

- $\gamma$ and $\gamma^{\prime}$ intersect transversely, and if $t_{1}, t_{2}, t_{1}^{\prime}, t_{2}^{\prime} \in[0,1]$ satisfy the property that $\gamma\left(\left[t_{1}, t_{2}\right]\right) \cup \gamma^{\prime}\left(\left[t_{1}^{\prime}, t_{2}^{\prime}\right]\right)$ bounds an imbedded disk $A \subset D_{n}$, then $A \cap\left\{p_{1}, \ldots, p_{n}\right\} \neq \emptyset$ (i.e., $\gamma$ and $\gamma^{\prime}$ are transverse and form no empty bigons).

Note that if $\gamma, \gamma^{\prime}$ are admissible arcs, there exist admissible arcs $\delta, \delta^{\prime}$ isotopic to $\gamma, \gamma^{\prime}$, resp., such that $\delta, \delta^{\prime}$ are pulled tight (cf. [11, Sec. 6]).

Definition 3.3. Let $\gamma, \gamma^{\prime}$ be two admissible arcs in $D_{n}$. We say $\gamma$ is right of $\gamma^{\prime}$ if, when pulled tight via isotopy, the orientation induced by the tangent vectors $\left.\frac{d \gamma}{d t}\right|_{t=0},\left.\frac{d \gamma^{\prime}}{d t}\right|_{t=0}$ agrees with the standard orientation on $D \subset \mathbb{C}$.

\footnotetext{
${ }^{3}$ If $\sigma$ is not a pure braid, which is easy to check by hand, then $\sigma \neq \mathbb{1}$.
} 


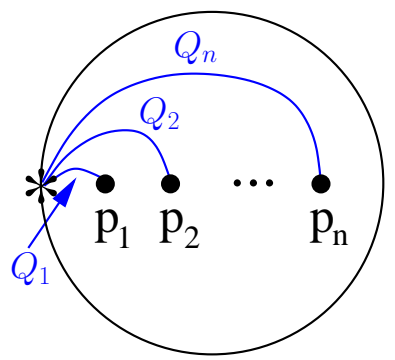

Figure 1. A collection of admissible $\operatorname{arcs} Q_{1}, \ldots, Q_{n} \subset D_{n}$ whose complement is a disk

There is a well-known isomorphism $\mathfrak{B}_{n} \cong \mathcal{M C G}\left(D_{n}\right)$ obtained by identifying the elementary Artin generator $\sigma_{i}$ (resp., $\sigma_{i}^{-1}$ ) with a homeomorphism of $D_{n}$ that acts as the identity outside of a small disk enclosing $p_{i}, p_{i+1}$ and acts as a $180^{\circ} \mathrm{CCW}$ (resp., CW) rotation on a (slightly smaller) disk enclosing $p_{i}, p_{i+1}$. Note that when a braid is viewed as a mapping class, it acts on $D_{n}$ on the right (since Artin braids are read from left to right).

Via the above isomorphism, $\mathfrak{B}_{n}$ acts on the set of isotopy classes of admissible arcs. Let $(\gamma) \sigma$ denote the image of (the isotopy class of) $\gamma$ under $\sigma \in \mathfrak{B}_{n}$.

Remark 3.1. Note that in [11] (cf. Prop. 1.1.3), positive (resp., negative) Artin generators are identified with CW (resp., CCW) rotations so that braids may act on $D_{n}$ on the left. We chose our convention to match those in [20] and [3].

Definition 3.4. Let $\sigma \in \mathfrak{B}_{n}$. We say $\sigma$ is right-veering if, for all admissible $\operatorname{arcs} \gamma,(\gamma) \sigma$ is right of $\gamma$ when pulled tight.

Remark 3.2. We may analogously define the notion of left-veering. A braid $\sigma \in \mathfrak{B}_{n}$ is left-veering (resp. right-veering) iff its mirror $m(\sigma) \in \mathfrak{B}_{n}$ is right-veering (resp. left-veering).

The following lemma is well-known:

Lemma 3.1. If $\sigma \in \mathfrak{B}_{n}$ is both right-veering and left-veering, then $\sigma=\mathbb{1}$.

Proof. If $\sigma \in \mathfrak{B}_{n}$ is both right- and left-veering, it must send each admissible arc to an isotopic admissible arc. Consider the collection $\left\{Q_{1}, \ldots, Q_{n}\right\}$ of admissible arcs pictured in Figure 1. A straightforward inductive argument then shows that $\sigma$ is isotopic to a map which fixes each $Q_{i}$ in this collection. The Alexander lemma (cf. [13, Lem. 2.1]) then implies that $\sigma=\mathbb{1}$, as desired.

Let $\psi(\widehat{\sigma}) \in \operatorname{Kh}(\widehat{\sigma})$ denote Plamenevskaya's invariant [39] of the transverse link in the tight contact structure on $S^{3}$ represented by the braid $\widehat{\sigma}$.

Proposition 3.1. If $\sigma$ is not right-veering, then $\psi(\widehat{\sigma})=0$.

Proof. Suppose $\sigma$ is not right-veering. Then there exists some admissible arc $\gamma$ which is sent to the left by $\sigma$. Let $\tau \in \mathfrak{B}_{n}$ satisfy $\gamma=\left(Q_{1}\right) \tau$. Then $\left(Q_{1}\right) \tau \sigma \tau^{-1}$ is left of $Q_{1}$. By [11. Prop. 6.2.7], there exists a $\sigma_{i}$-negative word (111, Defn. 1.2.3]) representing $\tau \sigma \tau^{-1}$ for some $i \in\{1, \ldots, n\}$. Such a word contains at least one letter $\sigma_{i}^{-1}$ and no letters $\sigma_{i}$. It then follows from [39, Proposition 3] that $\psi\left(\widehat{\tau \sigma \tau^{-1}}\right)=\psi(\widehat{\sigma})=0$. 
Corollary 1. If $\psi(\widehat{\sigma}) \neq 0$ and $\psi(m(\widehat{\sigma})) \neq 0$, then $\sigma=\mathbb{1}$.

Proof. By Proposition 3.1, $\sigma$ is both right- and left-veering. By Lemma 3.1, $\sigma=\mathbb{1}$.

\subsection{Proof of Theorem 1 .}

Proof of Theorem 1. (a). Suppose $\operatorname{SKh}(\widehat{\sigma}) \cong \operatorname{SKh}(\widehat{\mathbb{1}})$. According to Roberts 40, the invariant $\psi(\widehat{\sigma})$ is the image of the generator of the bottommost $k$ grading of $\operatorname{SKh}(\widehat{\sigma})$ under the spectral sequence from $\operatorname{SKh}(\widehat{\sigma})$ to $\operatorname{Kh}(\widehat{\sigma})$. By direct computation, $\operatorname{SKh}(\widehat{\sigma})$ is supported in a single homological grading, which implies that the spectral sequence from $\operatorname{SKh}(\widehat{\sigma})$ to $\mathrm{Kh}(\widehat{\sigma})$ collapses immediately. It follows that $\psi(\widehat{\sigma}) \neq 0$. The symmetry of sutured Khovanov homology under taking mirrors [40, Lem. 2] implies that $\operatorname{SKh}(m(\widehat{\sigma}))$ is also supported in a single homological grading. It follows that $\psi(m(\widehat{\sigma})) \neq 0$ as well. Corollary 1 then tells us that $\sigma=\mathbb{1}$, as desired.

The proof of Theorem 1.(b) is very similar in spirit to that of Theorem 1.(a). The analogue of Proposition 3.1 was proved in [3].

Proof of Theorem 1.(b). For $\sigma \in B_{n}$, Baldwin, Vela-Vick and Vértesi define in [3] a class $\widehat{t}(\widehat{\sigma}) \in \widehat{\mathrm{HFL}}(m(\widehat{\sigma}))$ which is an invariant of the transverse link represented by $\widehat{\sigma}$ (and agrees, for transverse knots, with the invariants defined in [38, 27]). We show below that if $\sigma$ is a pure braid and $\widehat{\mathrm{HFL}}(\widehat{\sigma} \cup B) \cong \widehat{\mathrm{HFL}}(\widehat{\mathbb{1}} \cup B)$, then $\widehat{t}(\widehat{\sigma}) \neq 0$ and $\widehat{t}\left(\widehat{\sigma^{-1}}\right) \neq 0$. An analogue of Corollary 1 then implies that $\sigma$ is equal to $\mathbb{1}$.

Suppose $\sigma$ is a pure braid and $\widehat{\mathrm{HFL}}(\widehat{\sigma} \cup B) \cong \widehat{\mathrm{HFL}}(\widehat{\mathbb{1}} \cup B)$. We first compute the latter. Let us denote $\mathbb{1}$ by $\mathbb{1}_{n}$ to indicate that it is the trivial braid on $n$-strands. Note that $\widehat{\mathbb{1}}_{n} \cup B$ is isotopic to link gotten by taking the connected sum of the positive Hopf link $\widehat{\mathbb{1}}_{1} \cup B$ with itself $n$ times along the component B. As computed in [37, Sec. 12] (cf. also [29, Sec. 4]),

$$
\widehat{\operatorname{HFL}}\left(\widehat{\mathbb{1}}_{1} \cup B\right) \cong\left(V_{1} \otimes V_{2}\right)[1 / 2,1 / 2] .
$$

Here, $V_{i}$ is the triply-graded vector space $\mathbb{E} \oplus \mathbb{F}$ whose first and second summands are supported in Maslov gradings 0 and -1 and Alexander bi-gradings $(0,0)$ and $-e_{i}$, where $e_{i}$ is the $i$ th standard basis vector in $\mathbb{R}^{2}$. The $[1 / 2,1 / 2]$ indicates that we have shifted the Alexander bi-grading by $(1 / 2,1 / 2)$. Our initial assumption and the Künneth formula in 37. imply that

$$
\widehat{\operatorname{HFL}}(\widehat{\sigma} \cup B) \cong \widehat{\operatorname{HFL}}\left(\widehat{\mathbb{1}}_{n} \cup B\right) \cong \widehat{\operatorname{HFL}}\left(\widehat{\mathbb{1}}_{1} \cup B\right)^{\otimes n} \cong\left(V_{1}^{\otimes n} \otimes V_{2}^{\otimes n}\right)[n / 2, n / 2] .
$$

To compute $\widehat{t}(\widehat{\sigma})$, we need to know the link Floer homology of $m(\widehat{\sigma} \cup-B)$. From (4) together with the formulae in (2) and (3), it follows that

$$
\widehat{\operatorname{HFL}}(m(\widehat{\sigma} \cup-B)) \cong\left(V_{1}^{\otimes n} \otimes V_{2}^{\otimes n}\right)[n / 2, n / 2]
$$

as well. Lemma 2.2 then implies that there is a spectral sequence with $E_{1}$ term

$$
\widehat{\operatorname{HFL}}(m(\widehat{\sigma} \cup-B))[-n / 2,0] \cong\left(V_{1}^{\otimes n} \otimes V_{2}^{\otimes n}\right)[0, n / 2]
$$

and whose $E_{\infty}$ term (ignoring the $A_{m(-B)}$ grading) is isomorphic to $\widehat{\operatorname{HFL}}(m(\widehat{\sigma})) \otimes V$, where $V$ is the bi-graded vector space $\mathbb{F} \oplus \mathbb{F}$ whose summands are supported in Maslov gradings 0 
and -1 and $A_{m(\widehat{\sigma})}$ grading 0 . To reduce clutter, we introduce the notation

$$
\begin{aligned}
H(\widehat{\sigma}, B) & :=\widehat{\operatorname{HFL}}(m(\widehat{\sigma} \cup-B))[-n / 2,0], \\
H(\widehat{\sigma}) & :=\widehat{\operatorname{HFL}}(m(\widehat{\sigma})) \otimes V .
\end{aligned}
$$

Note that the top Maslov graded piece $H_{t o p}$ (in Maslov grading $-n$ ) of the portion of $H(\widehat{\sigma}, B)$ in the bottommost $A_{m(-B)}$ grading $-n / 2$ has rank one. The element $\widehat{t}(\widehat{\sigma})$ can be characterized in terms of the image in $H(\widehat{\sigma})$ of the generator of $H_{\text {top }}$ under the spectral sequence from $H(\widehat{\sigma}, B)$ to $H(\widehat{\sigma})$. In particular, $\widehat{t}(\widehat{\sigma})$ is non-zero iff $H_{\text {top }}$ survives under this spectral sequence [3, Corollary 6.9].

Note that $H(\widehat{\sigma}, B)$ is supported in $A_{m(\widehat{\sigma})}$ gradings $-n \leq A_{m(\widehat{\sigma})} \leq 0$. Since $H(\widehat{\sigma})$ is supported in $A_{m(\widehat{\sigma})}$ gradings that are symmetric about the origin (as discussed in Subsection 2.2 it must be that the portion of $H(\widehat{\sigma}, B)$ in negative $A_{m(\widehat{\sigma})}$ gradings dies in the spectral sequence. Moreover, the portion in $A_{m(\widehat{\sigma})}$ grading 0 has rank $2^{n}$. Therefore,

$$
\operatorname{rk}(H(\widehat{\sigma})) \leq 2^{n} .
$$

Now, Lemma 2.3 implies that there is a spectral sequence whose $E_{1}$ term is $H(\widehat{\sigma})$ and whose $E_{\infty}$ term is a rank $2^{|m(\widehat{\sigma})|}$ vector space. Since $\sigma$ is a pure braid, $|m(\widehat{\sigma})|=n$. Thus,

$$
\operatorname{rk}(H(\widehat{\sigma})) \geq 2^{n} .
$$

It follows that $\operatorname{rk}(H(\widehat{\sigma}))=2^{n}$. It must therefore be the case that the entire portion of $H(\widehat{\sigma}, B)$ in $A_{m(\widehat{\sigma})}$ grading 0 survives in the spectral sequence from $H(\widehat{\sigma}, B)$ to $H(\widehat{\sigma})$. Since $H_{\text {top }}$ is contained in this portion we may deduce that $\widehat{t}(\widehat{\sigma})$ is non-zero. Hence, $\sigma$ is rightveering by [3, Theorem 1.4].

Note that $\widehat{\sigma^{-1}} \cup B$ is isotopic to $m(-\widehat{\sigma} \cup B)$. The formulae in 22 and 3 therefore imply that

$$
\widehat{\mathrm{HFL}}\left(\widehat{\sigma^{-1}} \cup B\right) \cong \widehat{\mathrm{HFL}}(\widehat{\sigma} \cup B) \cong \widehat{\mathrm{HFL}}(\widehat{\mathbb{1}} \cup B)
$$

as well. It follows that $\widehat{t}\left(\widehat{\sigma^{-1}}\right)$ is also non-zero. As before, this implies that $\sigma^{-1}$ is rightveering and, hence, that $\sigma$ is left-veering. Since $\sigma$ is both right- and left-veering, it is equal to $\mathbb{1}$.

The invariants $\operatorname{SKh}(\widehat{\sigma})$ and $\widehat{\mathrm{HFL}}(\widehat{\sigma} \cup B)$ can be used to solve the word problem in $B_{n}$ as follows. Suppose $w$ and $w^{\prime}$ are words in the generators $\sigma_{1}, \ldots, \sigma_{n}$ and their inverses representing the braids $\sigma(w)$ and $\sigma\left(w^{\prime}\right)$ in $B_{n}$. Let $\sigma=\sigma(w) \cdot\left(\sigma\left(w^{\prime}\right)\right)^{-1}$ and note that

$$
\sigma=\mathbb{1} \Longleftrightarrow \sigma(w)=\sigma\left(w^{\prime}\right) .
$$

In particular, if $\sigma$ is not a pure braid, then $\sigma(w) \neq \sigma\left(w^{\prime}\right)$. If $\sigma$ is a pure braid, compute $\operatorname{SKh}(\widehat{\sigma})$ or $\widehat{\mathrm{HFL}}(\widehat{\sigma} \cup B)$ (which can be done combinatorially) and apply Theorem 1 with $(5)$ in mind.

\section{Transverse Mirror Invariance: No Solution To Conjugacy Problem}

In this section, we show that $\operatorname{SKh}(\widehat{\sigma})$ and $\widehat{\operatorname{HFL}}(\widehat{\sigma} \cup B)$ cannot always distinguish nonconjugate braids. This fact is made precise in Corollary 2 of Theorem 2 below. First, some definitions and remarks.

Definition 4.1. Let $\sigma \in B_{n}$ and suppose $\sigma=\sigma(w)$ for some word $w$ in the generators $\sigma_{1}, \ldots, \sigma_{n}$ and their inverses. Then the reverse of $\sigma$ is the braid $\sigma^{r}=\sigma\left(w^{r}\right)$, where $w^{r}$ is the word obtained from $w$ by reversing the order of its letters. 

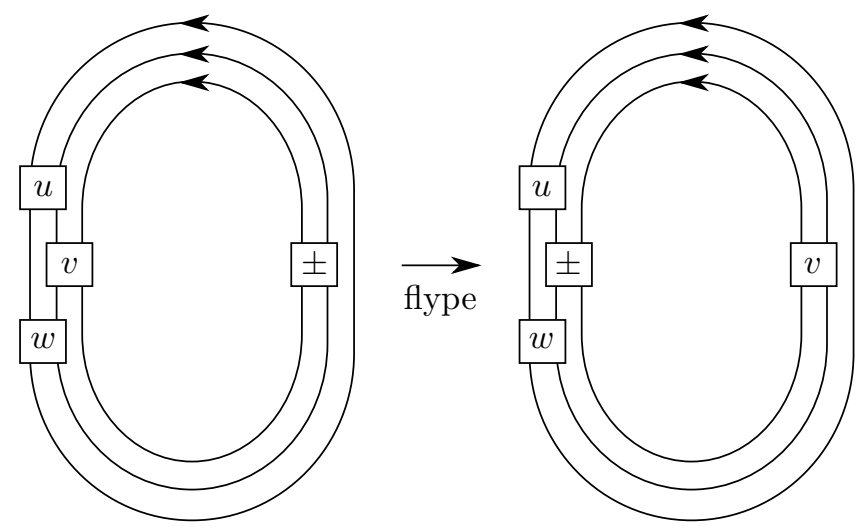

Figure 2. Left, a 3-braid closure $\widehat{\sigma}$. Each of the boxes labeled $u, v, w$ represents some number of positive or negative half twists. The box labeled \pm represents a single positive or negative half twist. Right, the closed braid obtained from $\widehat{\sigma}$ by a flype. It is oriented isotopic to $\widehat{\sigma}^{r}$ in $A \times I$.

Remark 4.1. Thought of as transverse links in the tight contact structure on $S^{3}$, the closure $\widehat{\sigma}^{r}$ is the transverse mirror of $\widehat{\sigma}$, as defined by $\mathrm{Ng}$ in [33, Defn. 4.5].

Theorem 2. Suppose $\sigma \in B_{n}$. Then

(a) $\operatorname{SKh}(\widehat{\sigma}) \cong \operatorname{SKh}\left(\widehat{\sigma}^{r}\right)$,

(b) $\widehat{H F L}(\widehat{\sigma} \cup B) \cong \widehat{H F L}\left(\widehat{\sigma}^{r} \cup B\right)$.

Proof of Theorem 2. (a). Let $D$ and $D^{r}$ denote the projections of $\widehat{\sigma}$ and $\widehat{\sigma}^{r}$ onto $A \times\{1 / 2\}$ as described in Subsection 2.1. There is an obvious bijection between oriented resolutions of $D$ and $D^{r}$ which preserves the $(i, j, k)$ triple-grading and commutes with the differentials in the complexes $\operatorname{CKh}(D)$ and $\operatorname{CKh}\left(D^{r}\right)$. (The projection $D^{r}$ is exactly what you see by looking at $D$ from below and reversing orientations.) It follows that $\operatorname{SKh}(\widehat{\sigma}) \cong \operatorname{SKh}\left(\widehat{\sigma}^{r}\right)$.

Proof of Theorem 2.(b). Note that if $\mathbb{L}=\widehat{\sigma} \cup B$, then $\widehat{\sigma}^{r} \cup B$ is oriented isotopic to $-\mathbb{L}$ in $S^{3}$. It follows from $\sqrt[1]{1}$ and $(2)$ that $\widehat{\mathrm{HFL}}(\mathbb{L}) \cong \widehat{\mathrm{HFL}}(-\mathbb{L})$ for any oriented link $\mathbb{L}$.

Corollary 2. There exist infinitely many pairs $\left(\sigma, \sigma^{\prime}\right) \in B_{3} \times B_{3}$ such that $\sigma \nsim \sigma^{\prime}$ but

$$
\operatorname{SKh}(\widehat{\sigma}) \cong \operatorname{SKh}\left(\widehat{\sigma}^{\prime}\right) \text { and } \widehat{H F L}(\widehat{\sigma} \cup B) \cong \widehat{H F L}\left(\widehat{\sigma}^{\prime} \cup B\right) .
$$

Proof. Suppose $\sigma$ and $\sigma^{\prime}$ are 3-braids for which $\widehat{\sigma}$ and $\widehat{\sigma}^{\prime}$ are related by a flype, as described in [9, Fig. 1.2] and illustrated in Figure 2. Then $\widehat{\sigma}^{\prime}$ is clearly oriented isotopic to $\widehat{\sigma}^{r}$ in $A \times I$. In particular, $\widehat{\sigma}^{\prime}$ and $\widehat{\sigma}$ are transverse mirrors, a fact first observed by $\mathrm{Ng}$ in 33 . Theorem 2 then implies that

$$
\operatorname{SKh}(\widehat{\sigma}) \cong \operatorname{SKh}\left(\widehat{\sigma}^{\prime}\right) \text { and } \widehat{\operatorname{HFL}}(\widehat{\sigma} \cup B) \cong \widehat{\operatorname{HFL}}\left(\widehat{\sigma}^{\prime} \cup B\right) .
$$

On the other hand, Birman and Menasco have enumerated infinitely many conjugacy classes of 3-braids admitting non-degenerate flypes; i.e. flypes which do not preserve conjugacy class [9, Tab. 2].

Remark 4.2. Let $H(\widehat{\sigma})$ denote either of the theories $\operatorname{SKh}(\widehat{\sigma})$ or $\widehat{\mathrm{HFL}}(\widehat{\sigma} \cup B)$. Given Theorem 2 and Corollary 2, one wonders, for braids $\sigma, \tau$ in $B_{n}$, whether $H(\widehat{\sigma}) \cong H(\widehat{\tau})$ implies that 
$\sigma \sim \tau$ or $\sigma \sim \tau^{r}$. This is almost certainly too optimistic. It is more reasonable to ask the following.

Question 4.1. Does $H\left(\widehat{\sigma}^{k}\right) \cong H\left(\widehat{\tau}^{k}\right)$ for all integers $k \geq 0$ imply that $\sigma \sim \tau$ or $\sigma \sim \tau^{r}$ ? What if we also assume that $\sigma$ and $\tau$ are alternating braids?

This question is motivated to some extent by work of Gonzáles-Meneses, who proves in 14] that if $\sigma^{k}=\tau^{k}$ for some $k$ then $\sigma \sim \tau$. The notion that the answer to Question 4.1 might be "yes" for alternating braids is inspired by Greene's recent result that if $D_{1}, D_{2}$ are reduced, alternating planar diagrams for knots $K_{1}, K_{2}$ in $S^{3}$ and $\widehat{\mathrm{HF}}\left(\Sigma\left(S^{3}, K_{1}\right)\right) \cong$ $\widehat{\mathrm{HF}}\left(\Sigma\left(S^{3}, K_{2}\right)\right)$, then $D_{1}, D_{2}$ are related by a sequence of Conway mutations [15].

\section{REFERENCES}

[1] M. M. Asaeda, J. H. Przytycki, and A. S. Sikora. Categorification of the Kauffman bracket skein module of I-bundles over surfaces. Algebr. Geom. Topol., 4:1177-1210 (electronic), 2004.

[2] D. Auroux, J. E. Grigsby, and S. M. Wehrli. Sutured Khovanov homology, Hochschild homology, and the Ozsváth-Szabó spectral sequence. math.GT/1303.1986, 2013.

[3] John A Baldwin, David Vela-Vick, and Vera Vértesi. On the equivalence of Legendrian and transverse invariants in knot Floer homology. Geom. Topol., 17(2):925-974, 2013.

[4] J. Batson and C. Seed. A link splitting spectral sequence in Khovanov homology. math.GT/1303.6240, 2013.

[5] S. J. Bigelow. The Burau representation is not faithful for $n=5$. Geom. Topol., 3:397-404 (electronic), 1999.

[6] S. J. Bigelow. Braid groups are linear. J. Amer. Math. Soc., 14(2):471-486 (electronic), 2001.

[7] J. S. Birman and T. E. Brendle. Braids: a survey. In Handbook of knot theory, pages 19-103. Elsevier B. V., Amsterdam, 2005.

[8] J. S. Birman and W. W. Menasco. Studying links via closed braids. V. The unlink. Trans. Amer. Math. Soc., 329(2):585-606, 1992.

[9] J. S. Birman and W. W. Menasco. A note on closed 3-braids. Commun. Contemp. Math., 10(suppl. 1):1033-1047, 2008.

[10] J.C. Cha and C. Livingston. Knotinfo table of knot invariants, 2006. Available from http://www. indiana.edu/ knotinfo/

[11] Patrick Dehornoy, Ivan Dynnikov, Dale Rolfsen, and Bert Wiest. Why are braids orderable?, volume 14 of Panoramas et Synthèses [Panoramas and Syntheses]. Société Mathématique de France, Paris, 2002.

[12] Alan Diaz. Plamenevskaya's transverse invariant and the self-linking number. To appear, 2013.

[13] B. Farb and D. Margalit. A primer on mapping class groups, volume 49 of Princeton Mathematical Series. Princeton University Press, Princeton, NJ, 2012.

[14] Juan González-Meneses. The $n$th root of a braid is unique up to conjugacy. Algebr. Geom. Topol., 3:1103-1118 (electronic), 2003.

[15] Joshua Evan Greene. Lattices, graphs, and Conway mutation. Invent. Math., 192(3):717-750, 2013.

[16] J. E. Grigsby and S. M. Wehrli. Khovanov homology, sutured Floer homology and annular links. Algebr. Geom. Topol., 10(4):2009-2039, 2010.

[17] J. E. Grigsby and S. M. Wehrli. On gradings in Khovanov homology and sutured Floer homology. In Topology and geometry in dimension three, volume 560 of Contemp. Math., pages 111-128. Amer. Math. Soc., Providence, RI, 2011.

[18] M. Hedden and Y. Ni. Khovanov module and the detection of unlinks. math.GT/1204.0960, 2012.

[19] M. Hedden and L. Watson. On the geography and botany of knot Floer homology. To appear, 2013.

[20] K. Honda, W. H. Kazez, and G. Matić. Right-veering diffeomorphisms of compact surfaces with boundary. Invent. Math., 169(2):427-449, 2007.

[21] M. Khovanov and P. Seidel. Quivers, Floer cohomology, and braid group actions. J. Amer. Math. Soc., 15(1):203-271 (electronic), 2002.

[22] D. Krammer. Braid groups are linear. Ann. of Math. (2), 155(1):131-156, 2002.

[23] P. B. Kronheimer and T. S. Mrowka. Khovanov homology is an unknot-detector. Publ. Math. Inst. Hautes Études Sci., (113):97-208, 2011.

[24] R. J. Lawrence. Homological representations of the Hecke algebra. Comm. Math. Phys., 135(1):141-191, 1990. 
[25] R. Lipshitz, P. Ozsváth, and D. Thurston. Bimodules in bordered Heegaard Floer homology. math.GT/1003.0598, 2010.

[26] R. Lipshitz, P. Ozsváth , and D. P. Thurston. A faithful linear-categorical action of the mapping class group of a surface with boundary. math.GT/1012.1032, 2010.

[27] Paolo Lisca, Peter Ozsváth, András I. Stipsicz, and Zoltán Szabó. Heegaard Floer invariants of Legendrian knots in contact three-manifolds. J. Eur. Math. Soc. (JEMS), 11(6):1307-1363, 2009.

[28] D.D. Long and M. Paton. The Burau representation is not faithful for $n \geq 6$. Topology, 32:439-447, 1993.

[29] C. Manolescu, P. Ozsváth, and S. Sarkar. A combinatorial description of knot Floer homology. Annals of Math., 169(2):633-660, 2009.

[30] Ciprian Manolescu, Peter Ozsváth, Zoltán Szabó, and Dylan Thurston. On combinatorial link Floer homology. Geom. Topol., 11:2339-2412, 2007.

[31] J. A. Moody. The Burau representation of the braid group $b_{n}$ is unfaithful for large $n$. Bull. Amer. Math. Soc., 25:379-384, 1991.

[32] H. R. Morton. The multivariable Alexander polynomial for a closed braid. In Low-dimensional topology (Funchal, 1998), volume 233 of Contemp. Math., pages 167-172. Amer. Math. Soc., Providence, RI, 1999.

[33] L. Ng. Combinatorial knot contact homology and transverse knots. Adv. Math., 227(6):2189-2219, 2011.

[34] Y. Ni. Homological actions on sutured Floer homology. math.GT/1010.2808, 2010.

[35] P. Ozsváth and Z. Szabó. Holomorphic disks and genus bounds. Geom. Topol., 8:311-334 (electronic), 2004.

[36] P. Ozsváth and Z. Szabó. Holomorphic disks and link invariants. math.GT/0512286, 2005.

[37] Peter Ozsváth and Zoltán Szabó. Holomorphic disks, link invariants and the multi-variable Alexander polynomial. Algebr. Geom. Topol., 8(2):615-692, 2008.

[38] Peter Ozsváth, Zoltán Szabó, and Dylan Thurston. Legendrian knots, transverse knots and combinatorial Floer homology. Geom. Topol., 12(2):941-980, 2008.

[39] O. Plamenevskaya. Transverse knots and Khovanov homology. Math. Res. Lett., 13(4):571-586, 2006.

[40] L. P. Roberts. On knot Floer homology in double branched covers. math.GT/0706.0741, 2007.

Boston College; Department of Mathematics; 301 Carney Hall; Chestnut Hill, Ma 02467

E-mail address: john.baldwin@bc.edu

Boston College; Department of Mathematics; 301 Carney Hall; Chestnut Hill, Ma 02467

E-mail address: julia.grigsby@bc.edu 